DOI: $10.5216 /$ cab.v12i1.8485

\title{
ECTOFAUNA PARASITÁRIA DE CÃES URBANOS DOMICILIADOS ATENDIDOS EM CLÍNICAS VETERINÁRIAS PARTICULARES NA CIDADE DE LAVRAS, MG
}

\author{
Antonio Marcos Guimarães, ${ }^{1}$ Bruno Sivieri Lima ${ }^{2}$ e Christiane Maria Barcellos Magalhães Rocha ${ }^{3}$ \\ 1. Professor associado de Doenças Parasitárias dos Animais Domésticos da Universidade Federal de Lavras (UFLA). E-mail: amg@ufla.br \\ 2. Médico veterinário formado pela UFLA e promotor de vendas do laboratório Vansil \\ 3. Professora adjunta de Epidemiologia Veterinária na UFLA.
}

\section{RESUMO}

O objetivo deste estudo foi determinar a frequência de ectoparasitos em cães urbanos domiciliados atendidos em nove clínicas veterinárias particulares do município de Lavras, MG. Espécimes de ectoparasitos, visíveis e palpáveis, foram coletados manualmente em 67 cães, durante o período de setembro de 2004 a maio de 2005. Posteriormente, foram acondicionados em frascos contendo etanol $70^{\circ}$ GL e identificados sob microscópio estereoscópio. No caso de ácaros causadores de sarna, efetuou-se a análise dos resultados de 155 raspados cutâneos obtidos de igual número de cães com suspeita clínica de dermatose parasitária, encaminhados para diagnóstico no Laboratório de Doenças Parasitárias da Universidade Federal de Lavras (UFLA), durante o período de setembro de 2002 a julho de 2007. Foi registrado um total de 540 ectoparasitos, sendo identificadas quatro espécies: Rhipicephalus sanguineus (60\%), Ctenocephalides canis e C. felis (ambas com $36 \%$ ) e larva de Dermatobia hominis (4\%). Nos raspados cutâneos, verificou-se positividade em $12,9 \%$ (20/155), dos quais $80 \%$ apresentavam Demodex canis (16/20) e 20\%, Sarcoptes scabiei var. canis (4/20). Após a identificação de ectoparasitos, foi montado um banco de dados com as fichas clínicas dos cães, analisado pelo programa SPSS 12.0. Pode-se concluir que as espécies de ectoparasitos predominantes em cães atendidos em clínicas veterinárias particulares do município de Lavras, MG foram Ctenocephalides canis e C. felis (Siphonaptera: Pulicidae), R. sanguineus (Acari: Ixodidae) e Demodex canis (Acari: Demodecidae), que teve um predomínio significativo $(\mathrm{p}<0,05)$ em raspados cutâneos de animais jovens suspeitos de demodicose canina.

PALAVRAS-CHAVES: Cães, ectoparasitos, Rhipicephalus sanguineus, Ctenocephalides, Demodex canis, Sarcoptes scabiei, larva de Dermatobia hominis.

\section{PARASITIC ECTOFAUNA OF URBAN DOMICILED DOGS EXAMINED IN PRIVATE VETERINARY CLINICS FROM LAVRAS MUNICIPALITY, MINAS GERAIS STATE, BRAZIL}

The objective of this study was to determine the frequency of ectoparasites in urban domiciled dogs treated at nine private veterinary clinics in the city of Lavras, Minas Gerais, Brazil. Visible and palpable ectoparasites specimens were collected manually from 67 dogs during the period of September 2004 to May 2005, kept in flasks with ethanol $70^{\circ} \mathrm{GL}$ and identified under a stereomicroscope. In the case of mites that cause mange, performed the analysis of the results obtained from 155 skin scrapings of the same number of dogs with clinical suspicion of parasitic dermatosis was performed, and sent to diagnostic at the Laboratory of Parasitic Diseases of Federal University of Lavras (UFLA), during the period of September 2002 to July 2007. A total of 540 ectoparasites were recorded, and four species were identified: Rhipicephalus sanguineus (60\%), Ctenocephalides canis and $C$. felis (both 36\%), and Dermatobia hominis larvae (4\%). In scraped skin, positive results were verified in $12.9 \%$ (20/155), of which $80.0 \%$ with Demodex canis (16/20) and $20.0 \%$ with Sarcoptes scabiei var. canis (4/20). After ectoparasites identification a 
database with the dogs'medical records was made and analyzed by SPSS 12.0. It can be concluded that the predominant species of ectoparasites in dogs treated at private veterinary clinics in the city of Lavras, MG, were Ctenocephalides canis and C.felis
(Siphonaptera: Pulicidae) and R. sanguineus (Acari: Ixodidae) and Demodex canis (Acari: Demodecidae) that had a significant predominance $(\mathrm{p}<0.05)$ on scraped skin of young animals with suspicion of canine demodicosis.

KEYWORDS: Dogs, ectoparasites, Rhipicephalus sanguineus, Ctenocephalides, Demodex canis, Sarcoptes scabiei, Dermatobia hominis larvae.

\section{INTRODUÇÃO}

Alguns artrópodes podem viver como ectoparasitos sobre os cães e expô-los a risco, assim como as pessoas que mantêm contato direto com os animais. A maioria desses parasitos pode transmitir doenças infecciosas e parasitárias tanto para os animais quanto para os seres humanos. (GONZÁLEZ et al., 2004).

Em cães infestados por sifonápteros do gênero Ctenocephalides sp (Siphonaptera: Pulicidae) e por Rhipicephalus sanguineus (Acari: Ixodidae) é comum o aparecimento da dermatite alérgica à picada de ectoparasitos - principalmente pulga (DAPP) - causa prurido, provoca desconforto nos animais e alterações dermatológicas secundárias decorrentes das picadas dos parasitos (KWOCHKA, 1987). Ctenocephalides felis é a espécie de pulga mais comum em cães e gatos na Europa, nos Estados Unidos (BECK et al., 2006) e no Brasil (BELLATO et al., 2003; TORRES et al., 2004), enquanto $C$. canis tem distribuição geográfica mais restrita e sua presença é assinalada principalmente em caninos (MARCHIONDO et al., 2007).

O parasitismo por $R$. sanguineus é muito comum entre os cães; esse carrapato é vetor biológico de Babesia canis, Ehrlichia canis, Hepatozoon canis, Mycoplasma haemocanis e Anaplasma platys (ALMOSNY, 2002), sendo a principal espécie observada em cães criados em áreas urbanas (LABRUNA \& PEREIRA, 2001). Ácaros causadores de sarna, como Demodex canis (Acari: Demodecidae) e Sarcoptes scabiei var. canis (Acari: Sarcoptidae), são responsáveis por dermatites parasitárias nos cães, podendo provocar alopecia, prurido, dermatite alérgica e pequenas lesões eritematosas (pápulas). $\mathrm{O}$ ato de coçar o local pode transformar a lesão em uma crosta, criando consequentemente, predisposição a infecções secundárias que podem causar piodermites de graus variados (LARSSON, 1995).
Levantamentos locais sobre a frequência de artrópodes ectoparasitos em cães são importantes tanto no âmbito da medicina veterinária como no da saúde pública, pois constituem a etapa inicial para a elaboração de medidas adequadas de controle e permitem avaliar o risco a que se expõem a população animal e os seres humanos. A ausência de informações sobre a ectofauna de parasitos em cães domiciliados, criados na área urbana do município de Lavras, MG, foi um dos principais motivos para a realização deste trabalho.

Em vista do exposto, com este estudo objetivou-se determinar a frequência de ectoparasitos em cães (Canis familiaris) domiciliados, criados na área urbana do município de Lavras, MG.

\section{MATERIAL E MÉTODOS}

Área de estudo

O município de Lavras está localizado na bacia do Rio Grande, na região sul de Minas Gerais, a 918 m de altitude ( $\left.21^{\circ} 14^{\prime} \mathrm{S}-45^{\circ} 00^{\prime}\right)$ e a $219 \mathrm{~km}$ da capital mineira. O município tem 87.421 habitantes, segundo dados do IBGE (2008). Na ausência de um censo específico, a população canina foi estimada em 12.488 animais. Utilizou-se para isso a população humana como indicador do tamanho da população canina urbana domiciliada, na proporção de um cão para cada sete habitantes na área urbana, de acordo com a recomendação feita pela Organização Mundial de Saúde (OMS) e adotada em campanhas de vacinação antirrábica em pequenos animais (WHO, 1990).

\section{Coletas e exames}

Para determinação da ectofauna, a coleta de ectoparasitos visíveis como carrapatos e pulgas foi realizada em 67 cães, no período de setembro de 2004 a maio de 2005. Para determinar a frequência de ectoparasitos causadores 
de sarna, examinaram-se 155 raspados cutâneos coletados de igual número de cães entre setembro de 2002 e julho de 2007.

As coletas de ectoparasitos visíveis foram realizadas independentemente do que havia motivado a ida do cão à clínica veterinária. No momento da coleta, o clínico veterinário preenchia uma ficha de identificação para cada animal, onde eram registrados dados relativos a sexo (macho ou fêmea), idade ( $\leq 1$ ou $>1$ ano), raça (definida ou mestiça), condição corporal (ruim, regular ou boa) e presença de lesões cutâneas (sim ou não). Alterações macroscópicas sugestivas de dermatoses foram identificadas observando-se sinais clínicos como lesões de pele dos tipos postular e escamosa, eritema e dermatite.

Os animais foram examinados minuciosamente para observação de ectoparasitos. Removeram-se as espécimes visíveis, como carrapatos e pulgas, manualmente. Elas foram armazenadas em pequenos frascos de plástico contendo etanol a 70\% GL, que após serem identificados individualmente permaneceram em temperatura ambiente até o momento da identificação dos parasitos. Larva de Dermatobia hominis (Diptera: Cuteribridae) também foi registrada, sempre que detectado sua presença.

Os espécimes de ectoparasitos visíveis foram coletados uma única vez sobre os cães domiciliados, de ambos os sexos, com diferentes idades e raças, atendidos em nove clínicas veterinárias particulares e criados na área urbana do município de Lavras, MG. Identificaram-se os espécimes coletados em microscópio binocular ou estereoscópico. Os carrapatos adultos foram classificados segundo FLECHTMANN (1973), enquanto os espécimes imaturos foram identificados genericamente. Classificaram-se as pulgas segundo a chave dicotômica proposta por LINARD \& GUIMARÃES (2000).

Para a determinação da frequência de ácaros causadores de sarna, foram analisados os resultados de raspados cutâneos obtidos de cães urbanos domiciliados e encaminhados por veterinários de clínicas particulares para o diagnóstico no Laboratório de Doenças Parasitárias do Departamento de Medicina Veterinária da Universidade Federal de Lavras (DMV/UFLA). Cada raspado cutâneo foi colocado sobre uma lâmina de vidro contendo uma gota de solução de hidróxido de potássio $(\mathrm{KOH})$ a $10 \%$. Posteriormente, foi coberto com uma lamínula e examinado ao microscópio com aumento de 100x e 400x para pesquisa de Demodex canis e Sarcoptes scabiei var. canis. Identificaram-se os ácaros causadores de sarna segundo MARCONDES (2001); o diagnóstico de demodicose foi confirmado quando observaram - se vários ácaros e/ou formas imaturas no raspado cutâneo.

\section{Análise estatística}

Após a identificação de ectoparasitos, foi montado um banco de dados com as fichas clínicas dos cães, analisado posteriormente pelo programa SPSS 12.0. Efetuou-se a distribuição de frequência de cada espécie de ectoparasito. Foram utilizados o teste do qui-quadrado ou teste exato de Fisher para determinar a relação entre idade, sexo e raça e a frequência dos ectoparasitos identificados, considerando-se um nível de confiança de $95 \%$.

\section{RESULTADOS E DISCUSSÃO}

Espécimes de ectoparasitos foram coletados em 67 cães - 49 de raça definida $(73,0 \%)$ e dezoito mestiços $(27,0 \%)$ - representados por 39 machos $(58,0 \%)$ e 28 fêmeas $(42,0 \%)$. As idades variaram entre dois meses e dez anos de vida, sendo $52,2 \%$ de jovens ( $\leq 1$ ano de idade) e $47,8 \%$ de adultos ( $>1$ ano de idade). No total foram coletados 540 ectoparasitos, identificados da seguinte forma: $60 \%$ de carrapatos (Rhipicephalus sanguineus); 36\% de pulgas (Ctenocephalides canis e C.felis) e 4\% de larva da mosca ou "berne" (Dermatobia hominis). Não houve diferença significativa $(\mathrm{p}>0,05)$ no nível de infestação de carrapatos e de pulgas de acordo com o sexo e a idade dos cães, demonstrando que tanto machos como fêmeas, jovens ou adultos, estão sujeitos a infestações por esses ectoparasitos. O nível de infestação por berne não foi analisado estatisticamente, devido à baixa frequência observada nos cães.

O número de espécies de artrópodes determinadas neste trabalho foi menor que o observado em outros estudos realizados no Brasil, nos quais se identificaram de cinco a sete espécies de ectoparasitos (RODRIGUES et al., 2001; BELLATO et al., 2003). Porém, carrapatos, seguidos de pulgas, também foram citados como os ectoparasitos mais frequentes nos 
estudos de TORRES et al. (2004), na região metropolitana de Recife, PE, e GONZÁLEZ et al. (2004), em Buenos Aires, Argentina. Entretanto, o predomínio de pulgas $(73,3 \%)$ em relação aos carrapatos $(63,3 \%)$ foi observado em 104 cães examinados em Juiz de Fora, MG (RODRIGUES et al., 2001).

Neste estudo a pequena variação de espécies se deve, provavelmente, ao fato de a população analisada ser constituída somente de cães urbanos domiciliados, atendidos em clínicas veterinárias particulares e que, consequentemente, recebiam mais cuidados. Essa hipótese é reforçada pela ausência de piolhos (anopluros ou malófagos) nos animais examinados, visto que a presença desses ectoparasitos evidencia certo grau de descuido com os cães.

Neste trabalho, $R$. sanguineus foi a única espécie de carrapato observada nos cães domiciliados criados na área urbana de Lavras, resultado que concorda com os de estudos realizados nos municípios de Porto Alegre, RS (RIBEIRO et al., 1997) e Juiz de Fora, MG (RODRIGUES et al., 2001; SOARES et al., 2006). Segundo LABRUNA\& PEREIRA(2001), R. sanguineus é o ixodídeo mais comum em cães criados em áreas urbanas. A intensidade da infestação não foi estimada no presente estudo, porém, constatou-se que entre os espécimes de $R$. sanguineus coletados predominaram os adultos. Esse fato pode ser parcialmente explicado pela forma como foi realizado o exame macroscópico pelos clínicos veterinários. Eles podem ter negligenciado a presença de larvas e ninfas, estádios parasitários de menor tamanho e, consequentemente, de maior dificuldade de visualização.

No presente estudo, Ctenocephalides sp foi o único gênero de pulicídeo identificado nos cães infestados, o que também constatou-se em outras pesquisas realizadas no Brasil (RODRIGUES et al., 2001; TORRES et al., 2004; SOARES et al., 2006), na Argentina (GONZÁLES et al., 2004) e na Itália (RINALDI et al., 2007). No município de Lavras, $C$. canis foi a espécie predominante, encontrada em 54\% dos cães (13/24), e C. felis foi observada em $29 \%$ dos animais (7/24). Infestações mistas foram assinaladas em $17 \%$ dos cães examinados (4/24). O fato de $C$. canis ser a espécie de pulga predominante em relação a $C$. felis diverge de estudos realizados no Brasil (TORRES et al., 2004; BELLATO et al., 2003), nos Estados Unidos (DURDEN et al., 2005) e na Itália (RINAL-
DI et al., 2007). Entretanto, esta pesquisa concorda com os trabalhos desenvolvidos no sul do Brasil por OLIVEIRA \& RIBEIRO (1982/1983). Esses autores sugerem que $C$. canis é uma espécie de sifonáptero que se adapta melhor a temperaturas mais baixas. $\mathrm{O}$ município de Lavras apresenta temperatura média baixa, principalmente nos meses de inverno, o que reforça a hipótese de a distribuição de C. canis estar relacionada principalmente a fatores climáticos como suspeitam OLIVEIRA \& RIBEIRO (1982/1983). Ainda de acordo com esses autores, é raro o encontrar $C$. canis fora do ambiente domiciliar. Afirmação que reforça os achados deste trabalho, cuja população analisada foi composta exclusivamente de cães urbanos domiciliados.

Em relação à sazonalidade, 96\% dos ectoparasitos (carrapatos e pulgas) foram observados entre os meses de setembro e janeiro (estação chuvosa). De acordo com GONZÁLEZ et al. (2004), R. sanguineus e Ctenocephalides sp preferem os meses quentes e úmidos, sendo, portanto, mais abundantes na primavera e no verão, com redução acentuada da população nos meses de outono e inverno.

Entre os cães envolvidos no presente estudo, 13\% apresentaram lesões macroscópicas (9/67), assim distribuídas: dermatite, 60\% (5/9); eritema, 20\% (2/9); ambas, $20 \%$ (2/9). Em 75\% dos cães examinados predominou a condição corporal boa dos animais (50/67), e em $25 \%$ a regular (17/67). Estes resultados estão coerentes com o perfil da população analisada, constituída de animais urbanos domiciliados, que dispõem de cuidados dos proprietários e assistência veterinária periódica. Não houve associação significativa $(p>0,05)$ entre condição corporal e lesões macroscópicas na pele no tocante à infestação dos animais por pulgas e carrapatos.

Entre os raspados cutâneos de cães, encaminhados ao Laboratório de Doenças Parasitárias do DMV/ UFLA, $16,1 \%$ foram positivos (25/155), sendo 81 de fêmeas $(52,0 \%), 68$ de machos $(44,0 \%)$ e seis de sexo indeterminado $(4,0 \%)$ na ficha individual. Desses positivos, 80\% apresentaram Demodex canis $(20 / 25)$ e $20 \%$, Sarcoptes scabiei var. canis (5/25). Estudos prévios realizados na Índia (NAYAK et al., 1997) e no México (RODRIGUEZ-VIVAS et al., 2003) mencionaram o $D$. canis como o principal ácaro causador de sarna em cães, seguido de $S$. scabei var. canis. Entretanto, pesquisas realizadas no Brasil não observaram 
diferença significativa ( $>00,05)$ nas frequências de $D$. canis e $S$. scabiei var. canis (BELLATO et al., 2003; TORRES et al., 2004). A frequência de $D$. canis de 12,9\% (20/155), observada em Lavras, MG, ficou muito acima das relatadas por BELLATO et al. (2003) em Lages, SC (1,96\%), e por TORRES et al. (2004) na região metropolitana de Recife, PE $(0,92 \%)$. Esse resultado deve-se, provavelmente, ao fato de a amostra do estudo em questão ser constituída exclusivamente de raspados cutâneos de cães com suspeita clínica de sarna, ou seja, animais com lesão sugestiva de dermatose parasitária. Porém, o índice aqui encontrado ficou abaixo do valor de $23 \%$ relatado por RODRIGUES-VIVAS et al. (2003) em Yacután, México.

A frequência de $D$. canis segundo a faixa etária, o sexo e a raça dos cães atendidos em clínicas veterinárias particulares do município de Lavras consta na Tabela 1. Houve diferença significativa $(p<0,05)$ entre frequência de $D$. canis e idade dos cães, sendo que em animais jovens ( $\leq 1$ ano de idade) há nove vezes mais chances de se encontrar esse demodecídeo que em adultos ( $>1$ ano de vida). Esse resultado concorda com os relatados em estudos anteriores (NAYAK et al., 1997; RODRIGUES-VIVAS et al., 2003) e pode ser explicado pela exposição constante dos cães jovens e com os sistemas imunológicos falhos às mães portadoras do ácaro $D$. canis.

Neste estudo não foi observada associação significativa ( $\mathrm{p}>0,05)$ alguma de raça e sexo com frequência de cães positivos para $D$. canis, sugerindo que caninos de diferentes raças, machos ou fêmeas, são igualmente suscetíveis a esse tipo de parasito. Esse resultado concorda com os encontrados por NAYAK et al. (1997) e RODRIGUEZ-VIVAS et al. (2003). A distribuição dos cães testados e dos casos de demodicose canina em função da idade, do sexo e da raça dos animais está representada na Tabela 1.

TABELA 1. Frequência (\%) de D. canis em 155 raspados cutâneos de cães urbanos domiciliados, examinados no Laboratório de Doenças Parasitárias do Departamento de Medicina Veterinária da Universidade Federal de Lavras (DMV/UFLA), MG no período de setembro de 2002 a julho de 2007

\begin{tabular}{|c|c|c|c|c|}
\hline Variável epidemiológica & № de cães examinados & № de cães positivos (\%) & Valor de $\mathrm{p}$ & $\begin{array}{c}\text { Odds ratio }(\mathrm{OR}) \\
\text { (Tendência) }\end{array}$ \\
\hline \multicolumn{5}{|l|}{ Idade (ano) } \\
\hline$\leq 1$ (jovem) & 54 & $13(24,1 \%)$ & $<0,05^{1}$ & $9,04(1,86 ; 85,29)$ \\
\hline$>1$ (adulto) & 59 & $2(3,3 \%)$ & & \\
\hline Indeterminada & 42 & $5(11,9 \%)$ & & \\
\hline \multicolumn{5}{|l|}{ Sexo } \\
\hline Fêmea & 81 & $10(12,3 \%)$ & $0,87^{2}$ & \\
\hline Macho & 68 & $9(13,2 \%)$ & & \\
\hline Indeterminado & 6 & $1(16,7 \%)$ & & \\
\hline \multicolumn{5}{|l|}{ Raça } \\
\hline Pura & 112 & $16(14,3 \%)$ & $0,78^{1}$ & \\
\hline Mestiça & 35 & $4(11,4 \%)$ & & \\
\hline Indeterminada & 8 & 0 & & \\
\hline Total & 155 & $20(12,9 \%)$ & & \\
\hline
\end{tabular}

${ }^{1}$ Teste exato de Fisher

${ }^{2}$ Teste do qui-quadrado.

\section{CONCLUSÃO}

Pode-se concluir que as pulgas Ctenocephalides canis e C.felis e o carrapato Rhipicephalus sanguineus constituem as espécies de ectoparasitos predominantes em cães urbanos domiciliados atendidos em clínicas veterinárias particulares do município de Lavras, MG. Nos casos de ácaros causadores de sarna, diagnostica- 
dos por meio de raspados cutâneos, houve um predomínio significativo de Demodex canis nos cães jovens $(\leq 1$ ano de idade) em relação aos animais adultos ( $>1$ ano de idade), suspeitos de demodicose canina.

\section{AGRADECIMENTO}

Aos clínicos veterinários da cidade de Lavras, MG por possibilitarem a realização deste estudo.

\section{REFERÊNCIAS}

ALMOSNY, N. R. P. Hemoparasitoses em pequenos animais domésticos e como zoonoses. Rio de Janeiro: L.F. Livros de Veterinária Ltda, 2002. 135 p.

BECK, W.; BOCH, K.; MACKENSEN, H.; WIEGAND, B.; PFISTER, K. Qualitative and quantitative observations on the flea population dynamics of dogs and cats in several areas of Germany. Veterinary Parasitology, v. 137, n. 1-2, p. 130-136, 2006.

BELLATO, V.; SARTOR, A. A.; SOUZA, A. P.; RAMOS, B. C. Ectoparasitas em caninos do município de Lages, Santa Catarina. Revista Brasileira de Parasitologia Veterinária, v. 12, n. 3, p. 95-98, 2003.

DURDEN, L.; JUDY, T. N.; MARTIN, J. E.; SPEDDING, L. S. Fleas parasitizing domestic dogs in Georgia, USA: species composition and seasonal abundance. Veterinary Parasitology, v. 130, n. 1-2, p. 157-162, 2005.

FLECHTMANN, C. H. W. Ácaros de importância médico veterinária. São Paulo: Nobel, 1973. 192 p.

GONZÁLEZ, A.; CASTRO, D. C.; GONZÁLEZ, S. Ectoparasitic species from Canis familiaris (Linné) in Buenos Aires province, Argentina. Veterinary Parasitology, v. 120, n. 1-2, p. 123-129, 2004.

IBGE. Instituto Brasileiro de Geografia e Estatística. Censo 2008. Disponível em: $<$ http://www.ibge.gov.br $>$. Acesso em: 5 nov. 2008.

KWOCHKA, K. W. Fleas and related disease. Veterinary Clinics of North America: Small Animal Practice, v. 17,n. 6,p. 1.235-1.260, 1987.

LABRUNA, M. B.; PEREIRA, M. C. Carrapato em cães. Clínica Veterinária, v. 6, p. 24-32, 2001.

LARSSON, C. E. Dermatoparasitoses de cães e gatos: patogenia, diagnóstico diferencial e saúde pública. Revista Brasileira de Parasitologia Veterinária, v. 4 (supl.1), n. 2, p. 261-270, 1995.

LINARDI, P. M.; GUIMARÃES, L. R. Sifonápteros do Brasil. São Paulo: Editora Museu de Zoologia USP/FAPESP, 2000. 291 p.
MARCHIONDO, A. A.; HOLDSWORTH, P. A.; GREEN, P.; BLAGBURN, B. L.; JACOBS, D. E. World Association for the Advancement of Veterinary Parasitology (W.A.A.V.P.) guidelines for evaluating the efficacy of parasiticides for the treatment, prevention and control of flea and tick infestation on dogs and cats. Veterinary Parasitology, v. 145, n. 1-2, p. 332-344, 2007.

MARCONDES, C. B. Entomologia médica e veterinária. São Paulo: Editora Atheneu, 2001. 432 p.

NAYAK, D. C.; TRIPATY, S. B.; DEY, P. C.; RAY, S. K.; MOHANTY, D. N.; PARIDA, G. S.; BISWAL, S.; DAS, M. Prevalence of canine demodicosis in Orissa (Índia). Veterinary Parasitology, v. 73, n. 3-4, p. 347-352, 1997.

OLIVEIRA, C. M. B.; RIBEIRO, P. B. Espécies de pulgas que parasitam cães em Porto Alegre e suas prevalências mensais. Arquivos da Faculdade de Veterinária UFRGS, v. 10-11, p. 29-33, 1982/1983.

RIBEIRO, V. L. S.; WEBER, M. A.; FETZER, L. O.; VARGAS, C. R. B. Espécies e prevalência das infestações por carrapatos em cães de rua da cidade de Porto Alegre, RS, Brasil. Ciência Rural, v. 27, n. 3, p. 285-289, 1997.

RINALDI, L.; SPERA, G.; MUSELLA, V.; CARBONE, S.; VENEZIANO, V.; IORI, A.; CRINGOLI, G. A survey of fleas on dogs in southern Italy. Veterinary Parasitology, v. 148, n. 3-4, p. $375-378,2007$.

RODRIGUES, A. F. S. F.; DAEMON, E.; D’AGOSTO, M. Investigação sobre alguns ectoparasitas em cães de rua no município de Juiz de Fora, Minas Gerais. Revista Brasileira Parasitologia Veterinária, v. 10, n. 1, p. 13-19, 2001.

RODRIGUEZ-VIVAS, R. I.; ORTEGA-PACHECO, A.; ROSADO-AGUILAR, J. A.; BOLIO, G. M. E. Factors affecting the prevalence of mange-mite infestations in stray dogs of Yucatán, Mexico. Veterinary Parasitology, v. 115, n. 1, p. 61-65, 2003.

SOARES, A. O.; SOUZA,A. D.; FELICIANO, E.A.; RODRIGUES, A. F. S. F.; D’AGOSTO, M.; DAEMON, M. Avaliação ectoparasitológica e hemoparasitológica em cães criados em apartamentos e casas com quintal na cidade de Juiz de Fora, MG. Revista Brasileira Parasitologia Veterinária, v. 15, n. 1, p. 13-16, 2006.

TORRES, F. D.; FIGUEIREDO, L. A.; FAUSTINO, M. A. G. Ectoparasitas de cães provenientes de alguns municípios da região metropolitana do Recife, Pernambuco, Brasil. Revista Brasileira Parasitologia Veterinária, v. 13, n. 4, p. 151-154, 2004.

WHO. World Health Organization. Guidelines for dog population management. Geneva: WHO, 1990. 